Document downloaded from:

http://hdl.handle.net/10251/166365

This paper must be cited as:

Chachques, JC.; Lila, N.; Soler Botija, C.; Martínez-Ramos, C.; Vallés Lluch, A.; Autret, G.; Perier, M.... (2020). Elastomeric cardiopatch scaffold for myocardial repair and ventricular support. European Journal of Cardio-Thoracic Surgery. 57(3):545-555. https://doi.org/10.1093/ejcts/ezz252

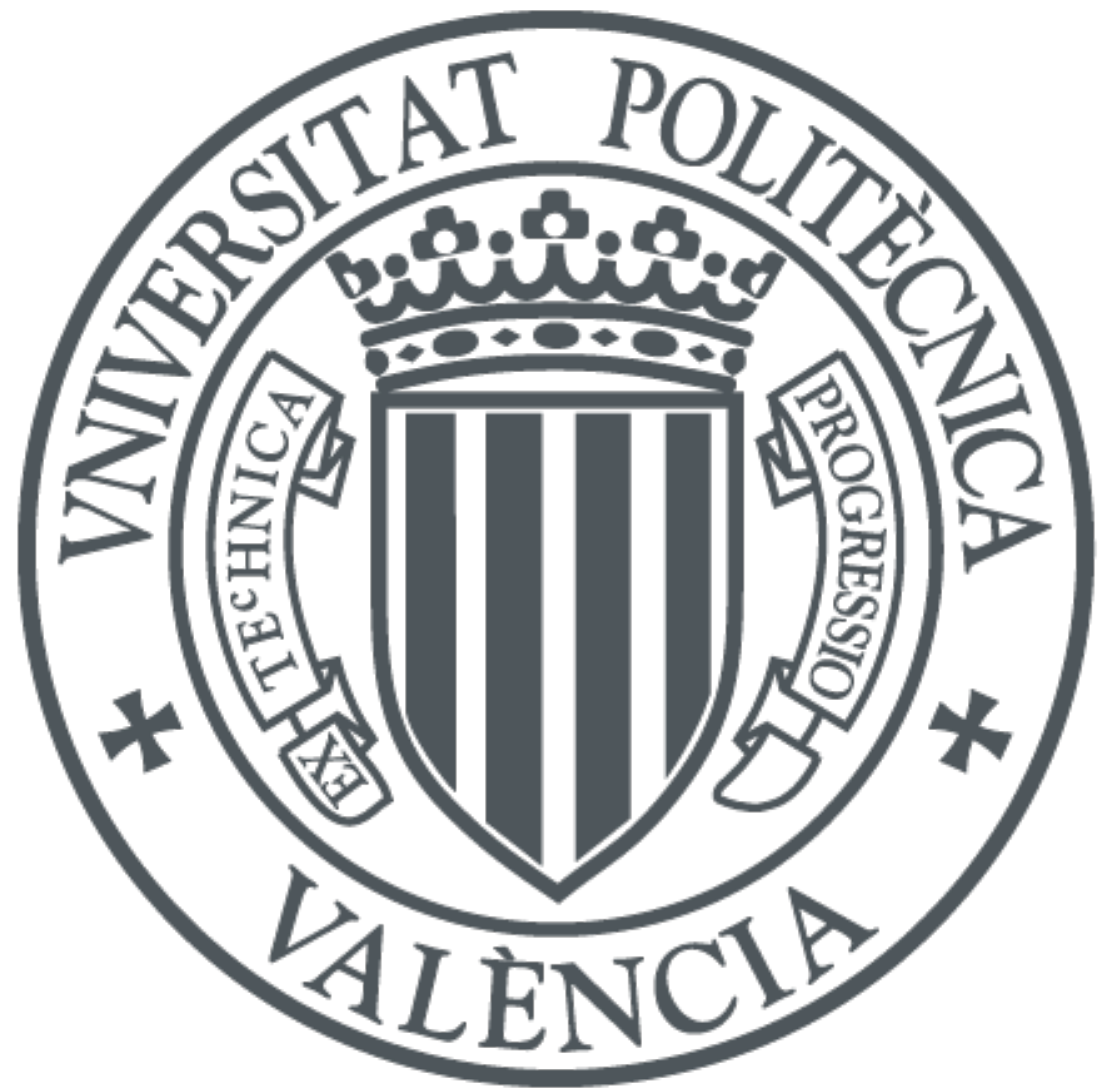

The final publication is available at

https://doi.org/10.1093/ejcts/ezz252

Copyright Oxford University Press

Additional Information 


\section{Elastomeric cardiopatch scaffold for myocardial repair and ventricular support}

Juan Carlos Chachques ${ }^{\mathrm{a},},{ }^{,}$Nermine Lila ${ }^{\mathrm{a}}$, Carolina Soler-Botija ${ }^{\mathrm{b}, \mathrm{c}}$, Cristina Martinez-Ramos ${ }^{\mathrm{d}}$, Ana Valles $^{d}$, Gwennhael Autret ${ }^{\mathrm{e}}$, Marie-Cecile Perier ${ }^{f}$, Nicolas Mirochnik ${ }^{\mathrm{g}}$, Manuel MonleonPradas $^{d}$, Antoni Bayes-Genis ${ }^{b}$ and Carlos E. Semino ${ }^{h}$

a Laboratory Biosurgical Research, Alain Carpentier Foundation, Cardiac Surgery Pompidou Hospital, University Paris-Descartes, Paris, France

b Research Cardiology Institute, Germans-Trias-Pujol Hospital, Badalona, Spain

c CIBER Cardiovascular, Carlos III Health Institute, Madrid, Spain

d Center for Biomaterials and Tissue Engineering, Polytechnic University Valencia, Valencia, Spain

e Microcirculation Imaging Lab, Paris Cardiovascular Research Center (PARCC), University Paris, Paris, France

f Cardiovascular Epidemiology Unit, PARCC, University Paris, Paris, France

g Cardiology Department, Pompidou Hospital, University Paris, Paris, France

h Bioengineering Department, IQS-School Engineering, Ramon-Llull University, Barcelona, Spain

* Corresponding author. Biosurgical Research Laboratory, Alain Carpentier Foundation, Cardiac Surgery Pompidou Hospital, 56 rue Leblanc, 75015 Paris, France. Tel: +33-613144398; fax: +33-156095903; e-mail: j.chachques@aphp.fr (J.C. Chachques)

Keywords: Heart failure, Cardiac tissue engineering, Elastomeric scaffold, Cardiopatch, Cardiowrap, Translational and clinical research

\section{Abstract}

OBJECTIVES: Prevention of postischaemic ventricular dilatation progressing towards pathological remodelling is necessary to decrease ventricular wall deterioration. Myocardial tissue engineering may play a therapeutic role due to its capacity to replace the extracellular matrix, thereby creating niches for cell homing. In this experimental animal study, a biomimetic cardiopatch was created with elastomeric scaffolds and nanotechnologies.

METHODS: In an experimental animal study in 18 sheep, a cardiopatch was created with adipose tissue-derived progenitor cells seeded into an engineered bioimplant consisting of 3dimensional bioabsorbable polycaprolactone scaffolds filled with a peptide hydrogel (PuraMatrixTM). This patch was then transplanted to cover infarcted myocardium. Nonabsorbable poly(ethyl) acrylate polymer scaffolds were used as controls.

RESULTS: Fifteen sheep were followed with ultrasound scans at 6 months, including echocardiography scans, tissue Doppler and spectral flow analysis and speckle-tracking imaging, which showed a reduction in longitudinal left ventricular deformation in the cardiopatchtreated group. Magnetic resonance imaging (late gadolinium enhancement) showed reduction of infarct size relative to left ventricular mass in the cardiopatch group versus the controls. Histopathological analysis at 6 months showed that the cardiopatch was fully anchored and integrated to the infarct area with minimal fibrosis interface, thereby promoting angiogenesis and migration of adipose tissue-derived progenitor cells to surrounding tissues.

CONCLUSIONS: This study shows the feasibility and effectiveness of a cardiopatch grafted onto myocardial infarction scars in an experimental animal model. This treatment decreased fibrosis, limited infarct scar expansion and reduced postischaemic ventricular deformity. A capillary network developed between our scaffold and the heart. The elastomeric cardiopatch seems to have a positive impact on ventricular remodelling and performance in patients with heart failure. 


\section{INTRODUCTION}

Extracellular matrix (ECM) remodelling in heart failure (excessive matrix degradation and myocardial fibrosis) contributes to left ventricular (LV) dilatation and progressive cardiac dysfunction. An appropriate balance of ECM synthesis and degradation is required for normal morphogenesis and maintenance of tissue architecture. In ischaemic heart disease, the imbalance in the ECM turnover either by decreased matrix synthesis and/or increased degradation leads to cardiac dilatation or infarct rupture [1-4]. Myocardial tissue engineering should provide structural support to the heart; specific scaffolds should help to normalize cardiac wall stress in injured regions by improving strain distribution $[1,5,6]$. Engineering materials requiring specific properties of stiffness and resistance to deformation can be implanted around the heart or seeded into the myocardial tissue. They are composed of a natural or synthetic structure capable of supporting 3-dimensional (3D) tissue formation. Scaffold characteristics are critical to recapitulating the in vivo milieu and allowing cells to influence their own microenvironments. Such scaffolds serve the following purposes: allow cell attachment and migration, deliver and retain cells and biochemical factors, enable diffusion of vital cell nutrients and expressed products and exert certain mechanical and biological influences to modify the behaviour of the cell phase $[1,5,7]$. The objective of this in vivo tissue engineering study was to assess the safety and efficacy of 3D scaffold cardiopatches in an myocardial infarction (MI) sheep model with pathophysiological and biomechanical degradation similar to that seen in humans. The cardiopatches were created using elastomeric porous membranes filled with nanometric self-assembling peptides seeded with autologous adipose tissue-derived progenitor cells (ATDPCs) and grafted onto LV MI scars.

\section{MATERIALS AND METHODS}

In 18 I^le-de-France sheep weighing $36.2 \pm 5.3 \mathrm{~kg}$, Mls were created with surgical occlusion of the left anterior descending diagonal coronary artery branches. Two months later, the MI was surgically treated either with semidegradable or with nondegradable cardiopatches made of elastomeric membranes filled with self-assembling peptide-RAD16-I PuramatrixTM (BD Biosciences, Franklin Lakes, NJ, USA) seeded with ATDPCs (Fig. 1A and B). A third control group had Mls without treatment. All experiments were performed at the Laboratory of Biosurgical Research (ISO-Certification 9001), Pompidou Hospital, University of Paris Descartes, Paris, France, and received care in compliance with the European Conventions. Animal studies were approved by the ethics committee for animal research of Paris Descartes University, France.

\section{Technique of experimental myocardial infarction}

Preoperative management, anaesthesia procedure and postoperative care were performed consistent with our previously described experimental studies [7]. A median sternotomy was performed, and the pericardial sac was opened exposing the heart. To reduce the risk of ventricular fibrillation, xylocaine 1\% (Lidocaine, AstraZeneca, Cambridge, UK) was continuously infused intravenously ( $2 \mathrm{mg} / \mathrm{kg}$ per hour) during the surgical procedure. Mediastinal fat tissue was harvested for isolation and expansion of ATDPCs. An MI was created by ligation of the diagonal branches of the left anterior descending coronary artery using 5-0 nonabsorbable Prolene sutures. Significant electrocardiographic changes, including widening of the QRS complex, elevation of the ST segment and colour and kinetic changes of the anterior wall were 
considered indicative of coronary occlusion. Transepicardial echocardiography was performed before and after the $\mathrm{Ml}$ to assess ventricular dimensions and function and to identify the infarcted area.

\section{Isolation, culture and transduction of adipose tissue-derived progenitor cells from sheep}

Cells were isolated from mediastinal adipose tissue by $0.05 \%$ collagenase II digestion at $37 \mathrm{C}$ and adhesion to the plate surface in a-minimum essential medium supplemented with $10 \%$ foetal bovine serum and 1\% penicillin-streptomycin. ATDPCs were cultured under standard conditions $(37 \mathrm{C}, 5 \% \mathrm{CO}$ ) (duplication time 0.7 days) and labelled with CMVp-RLuc-mRFP1 lentivirus (2 106 transduction units/ml; $\mathrm{MOI}=21$ ) for $48 \mathrm{~h}$. The highest $11 \%$ RFPexpressing cells were selected by FACS (Fluorescence-Activated Cell Sorting) to be used for implantation. Cells were then expanded to $100 \pm 7$ million and loaded into cardiopatch scaffolds.

\section{Preparation of cardiopatches}

Scaffold membranes consisted of a matrix with large-sized spherical pores $(130 \pm 20 \mathrm{~mm})$ with good interconnectivity obtained by a porogenic template-manufacturing technique (Fig. 1B). Sintered microbeads with a narrow distribution of diameters were used as a polymerization template and eliminated after polymerization of the matrix precursors in its interstices. The pores of the scaffolds were filled with PuraMatrix hydrogel $1 \%(w / v)$, which is capable of forming fibrillar structures in the range of nanometers [8]. Two different bioactive patches were developed: 1 . Semidegradable elastomeric porous membranes made from interpenetrating polymer networks of cross-linked hyaluronic acid and cross-linked polycaprolactone (CLMA). They are partially reabsorbed leaving a residual permanent light skeleton for mechanical support (Fig. 1) $[9,10]$. 2. Non-degradable elastomeric porous membranes from poly(- ethyl) acrylate (PEA) polymer filled with Puramatrix peptide hydrogel using a gentle vacuum.

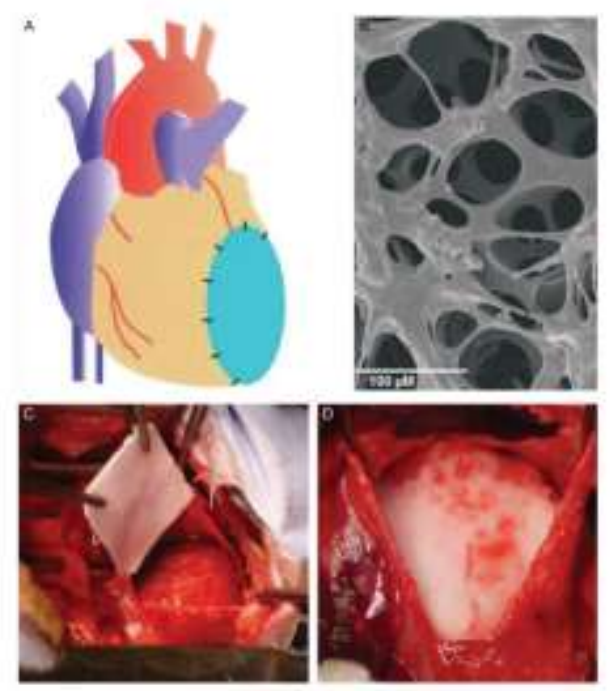

Figure 1: Elastomeric scaffolds for the treatment of chronic myocardial infarction. The cardiopatch is created with porous elastomers filled with nanometric peptides containing adipose tissue-derived progenitor cells ( $A$ and $B$ ). The cardiopatch was surgically grafted on sheep left ventricular infarct scars ( $C$ and $D)$. 


\section{Treatment with the cardiopatch (2months after infarct)}

General anaesthesia was readministered via the same protocol used for the MI model. The LV was accessed via a left thoracotomy through the 5th intercostal space.

Sheep were randomized into 3 treatment groups: group $1(n=5)$ had Mls without treatment (control group); group $2(n=5)$ had Mls treated with a CLMA patch; group $3(n=5)$ had MIs treated with the PEA patch.

\section{Implantation of cardiopatches}

The elastomeric membranes measured $5050 \mathrm{~mm}$ and were $2 \mathrm{~mm}$ thick; all were seeded with ATDPCs and placed onto the surface of the LV, then fixed to the epicardium with interrupted superficial sutures (Prolene 5-0 placed at the periphery of the cardiopatch) (Fig. 1).

\section{Evaluation at 6 months with echocardiography, magnetic resonance imaging and histopathological examination}

With the sheep under general anaesthesia, a redo sternotomy was performed. Transepicardial echocardiography was then carried out followed by magnetic resonance imaging (MRI) with an injection of an intravenous gadolinium solution at a dose of $0.4 \mathrm{ml} / \mathrm{kg}$. The animals were then sacrificed by a lethal intravenous injection of Dolethal (pentobarbital sodium $200 \mathrm{mg} / \mathrm{ml}$ ) (Vetoquinol, Lure, France). Transepicardial echocardiography A MyLab30-Gold cardiovascular ultrasound system (Esaote S.p.A., Firenze, Italy) equipped with a phased-array 1-5 MHz transducer was used to assess (i) LV end diastolic volume-end systolic volume, stroke volume, shortening fraction and ejection fraction; (ii) Doppler spectral flow for peak $E$ deceleration time, A-wave, LV outflow tract-velocity time integral; and (iii) tissue Doppler for peak velocities of the mitral annulus in early (EO ) phases of LV filling (measured at the lateral portion of the mitral annulus); the dimensionless ratio E/EO was computed. For each scan, 1 cardiac cycle was acquired at a frame rate of $60-70 \mathrm{~Hz}$. Deformation of the ventricular wall was evaluated by longitudinal strain using speckle-tracking based analysis [11].

\section{Magnetic resonance imaging}

A BioSpec 47/40 USR system (Bruker-Corp., Karlsruhe, Germany) was used for MRI studies. The extracellular MRI contrast medium gadolinium allowed detection of infarct scars by latehyperenhancement of the scar tissue. Immediately after autopsy, the isolated sheep hearts underwent ex vivo MRI to assess the infarct size (gadolinium white-marked areas) relative to myocardial mass and the integration of the cardiopatch onto the ventricular wall [12]. Threedimensional evaluations were performed using longitudinal and transverse MRI serial planes (Fig. 2).

\section{Histopathological studies}

All sheep were sacrificed at 6 months. The site of the myocardial injury was identified and dissected with 4-5 specimens taken and fixed in 10\% formalin, embedded in paraffin and sectioned into 10-Im-thick slices. The sections were stained with haematoxylin-eosin-saffron, Masson trichrome and Sirius red stains. A polarized light microscope was used to measure 
collagen types I and III on randomly selected Sirius red-stained sections from both infarct and border zones. To identify the grafted cells, sections were incubated with antibodies against cardiac troponin I and RFP (2 Ig/ml; Abcam, Cambridge, UK). Immunostaining against metalloproteinase 9 (MMP9; $10 \mathrm{lg} / \mathrm{ml}$; Chemokine Therapeutics Corp, Vancouver, BC, Canada) was also performed. Nuclei were counterstained with 40,6 -diamidino-2-phenylindole (Thermo Fisher Scientific, Waltham, MA, USA), and the results were analysed with an Axio-Observer Z1 (Zeiss, Oberkochen, Germany) laser confocal microscope. To determine vessel density, heart sections were stained with biotinylated GSLI B4 isolectin (Vector Laboratories, Burlingame, CA, USA), and Alexa 647-conjugated streptavidin (ThermoFisher). Images were taken in 10 randomly selected fields ( 5 infarcted areas +5 remote healthy areas) and analysed using ImageJ software (available at https://imagej.nih. gov/ij/). Results were expressed as a percentage of the mean isolectin-positive area per area of tissue surface.

\section{Statistical analysis}

Statistical analyses were performed with SPSS Statistics version 19 (IBM Corporation, Armonk, NY USA). Results were analysed and reported as percentage or mean \pm standard deviation or median interquartile range when appropriate. Differences between groups were compared using the Student's t-test or one-way analysis of variance for multiple comparisons. A P-value of $<0.05$ was considered to be statistically significant. Comparisons of echocardiography and MRI parameters across treatment groups were performed using analysis of variance and the non-parametric Kruskal-Wallis test.

\section{RESULTS}

There were 3 surgical complications and 1 animal died during the infarct induction procedure of irreversible ventricular fibrillation. Two animals were excluded from the study due to skin and mediastinal infections. In summary, 15 sheep survived (5 for each study group) and were evaluated over the long term.

\section{Echocardiography}

LV function and dimensions were quantified the day of the myocardial infarction. Only animals developing LV dysfunction with an ejection fraction less than $35 \%$ gained access to the second phase. At the 6-month follow-up examination, the attenuated parameters of ventricular deformation observed in the cardiopatch groups were assessed with speckle-tracking imaging (longitudinal strain). Diastolic function was improved (E deceleration time assessment) in treated groups versus controls. Significant benefits were also observed in the following ultrasound parameters: E/A ratio, E/EO ratio, LV outflow tract-velocity time integral (Table 1). 


\begin{tabular}{|c|c|c|c|c|c|c|c|}
\hline & \multicolumn{2}{|c|}{ Control } & \multicolumn{2}{|c|}{ CLMA } & \multicolumn{2}{|c|}{ PEA } & \multirow[b]{2}{*}{$P$-value ${ }^{a}$} \\
\hline & $n$ & $\begin{array}{l}\text { Mean (SD) or median } \\
\text { (interquartile range) }\end{array}$ & $n$ & $\begin{array}{l}\text { Mean (SD) or median } \\
\text { (interquartile range) }\end{array}$ & $n$ & $\begin{array}{l}\text { Mean (SD) or median } \\
\text { (interquartile range) }\end{array}$ & \\
\hline SL & 5 & $2.2 \pm 0.5$ & 5 & $4.7 \div 1.2$ & 5 & $7.3 \pm 1.6$ & 0.04 \\
\hline $\mathrm{DD}(\mathrm{mm})$ & 5 & $42.14 \pm 7.67$ & 5 & $43.33 \approx 4.76$ & 5 & $38.40 \pm 3.21$ & 0.37 \\
\hline SD (mm) & 5 & $31.00 \pm 6.48$ & 5 & $30.67 \pm 4.32$ & 5 & $25.80 \pm 3.27$ & 0.20 \\
\hline $\operatorname{EF}(\%)$ & 5 & $5200 \pm 9.06$ & 5 & $56.17 \pm 6.01$ & 5 & $61.40 \pm 9.79$ & 0.19 \\
\hline SF $(\%)$ & 5 & $26.71 \pm 5.62$ & 5 & $32.50 \pm 9.83$ & 5 & $32.80 \pm 6.91$ & 0.3 \\
\hline E/A ratio & 5 & $1.53 \pm 0.31$ & 5 & $1.35 \div 0.34$ & 5 & $1.04 \pm 0.32$ & 0.06 \\
\hline EDT (ms) & 5 & $118.14 \pm 23.2$ & 5 & $132.50=31.5$ & 5 & $147.80 \pm 27.42$ & 0.04 \\
\hline$E / E$ ratio & 5 & $5.10 \pm 1.3$ & 5 & $3.50 \pm 0.9$ & 5 & $4.30 \pm 1.2$ & 0.03 \\
\hline $\operatorname{vin}(\mathrm{cm})$ & 5 & $9.71 \pm 1.80$ & 5 & $12.00 \div 2.00$ & 5 & $11.2 \pm 1.9$ & 0.07 \\
\hline $\mathrm{SV}(\mathrm{mL})$ & 5 & $17.02 \pm 4.3$ & 5 & $23.79 \pm 5.21$ & 5 & $24.02 \pm 3.7$ & 0.28 \\
\hline
\end{tabular}

\section{Magnetic resonance imaging}

The white tonality of infarct scars is enhanced vividly $15 \mathrm{~min}$ after the administration of intravenous gadolinium, representing the accumulation of gadolinium in the extracellular space due to the loss of membrane integrity in the infarcted tissue. At the 6-month follow-up examination, CLMA patches were more adherent to and integrated into the ventricular wall compared with the PEA patches. The volume of the infarct scars related with the ventricular mass showed a significant reduction of the relative infarct volume in the cardiopatch-treated group, mainly in the CLMA group (Fig. 2; Table 2).
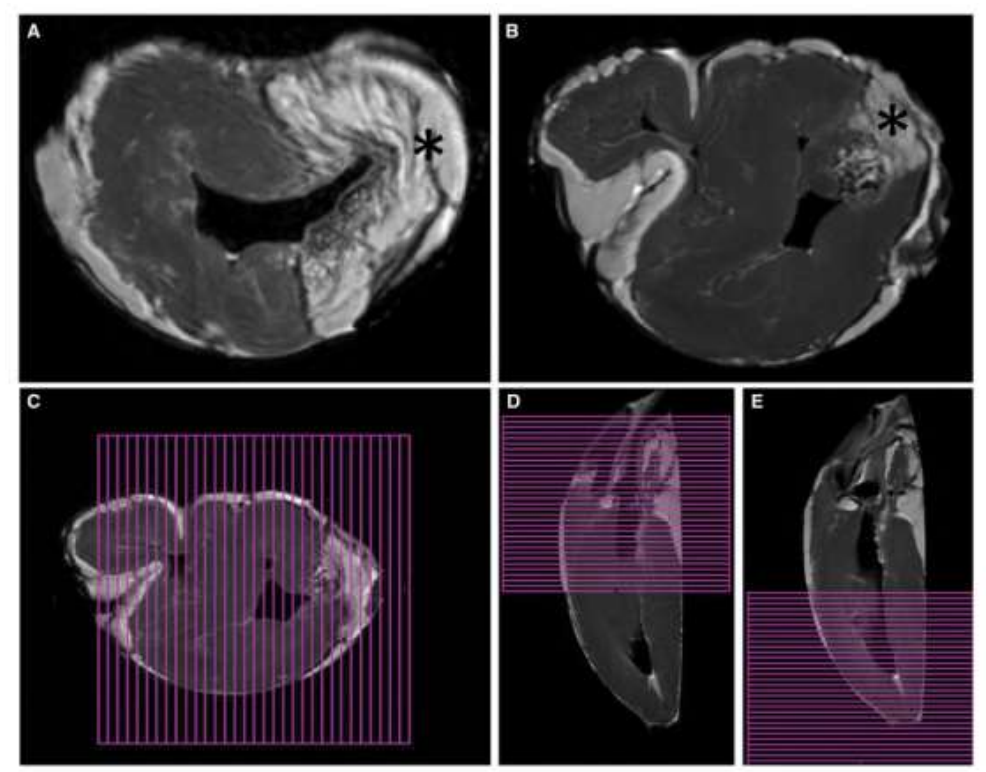

Figure 2: Cardiopatch evaluation with magnetic resonance imaging. Hyperenhancement on the infarct scar with the contrast medium gadolinium (signals in white). (A) Transverse left ventricular imaging showing large myocardial infarction (asterisk) in a control untreated sheep. (B) Magnetic resonance imaging showing reduced infarcted area (asterisk) in CLMA cardiopatch-treated sheep (both at 6-month follow-up). (C-E) Three-dimensional evaluation of myocardial mass and infarct size using transverse and longitudinal serial planes. 


\begin{tabular}{|c|c|c|c|}
\hline Group & $\begin{array}{l}\text { LV myocardial } \\
\text { mass, } \mathrm{cm}^{3}\end{array}$ & $\begin{array}{l}\text { 3-Dimensional } \\
\text { infarct size, } \mathrm{cm}^{3}\end{array}$ & $\begin{array}{l}\text { Infarct size/LV } \\
\text { myocardial mass, \% }\end{array}$ \\
\hline Control & $98.7 \pm 129$ & $13.8 \div 4.7$ & $13.9 \pm 2.3$ \\
\hline CLMA patch & $102.2 \pm 14.4$ & $6.4 \pm 1.6$ & $6.3 \pm 1.1^{2}$ \\
\hline PEA patch & $102.0 \pm 11.9$ & $10.1 \div 5.7$ & $9.9 \pm 4.8^{2}$ \\
\hline
\end{tabular}

\section{Left ventricle histopathology}

The surgical coronary artery ligation model for this study results in the transmural necrosis of the LV wall with central fibrosis and an intermediate border zone surrounded by healthy myocardium. In the cardiopatch-treated sheep, mainly in the CLMA group, the necrotic areas were significantly less prominent than in the non-treated control group (Fig. 3). To further investigate this observation, collagen content was measured in the infarcted regions and in the healthy myocardium bordering the infarcts. At sacrifice, the collagen volume fraction in the infarct core was $37.6 \pm 3.7 \%, 25.3 \pm 7.0 \%$ and $35.4 \pm 5.2 \%$ in the controls, the PEA and the CLMA-treated animals, respectively $(P=0.01)$. Collagen type I accounted for only $14.5 \%$ of ECM in the PEA-treated animals whereas it increased to $27.1 \%$ and $28.5 \%$ of total collagen in the controls and in the CLMA-treated group, respectively $(P=0.001)$. Collagen III contents did not present with statistical differences in any of the groups $(P=0.06)$. Collagen volume fraction values were also similar in the border regions with $4.7 \pm 3.5 \%, 2.1 \pm 1.1 \%$ and $2.9 \pm 2.0 \%$ in the controls and in the PEA- and CLMA-treated animals, respectively $(P=0.24)$.

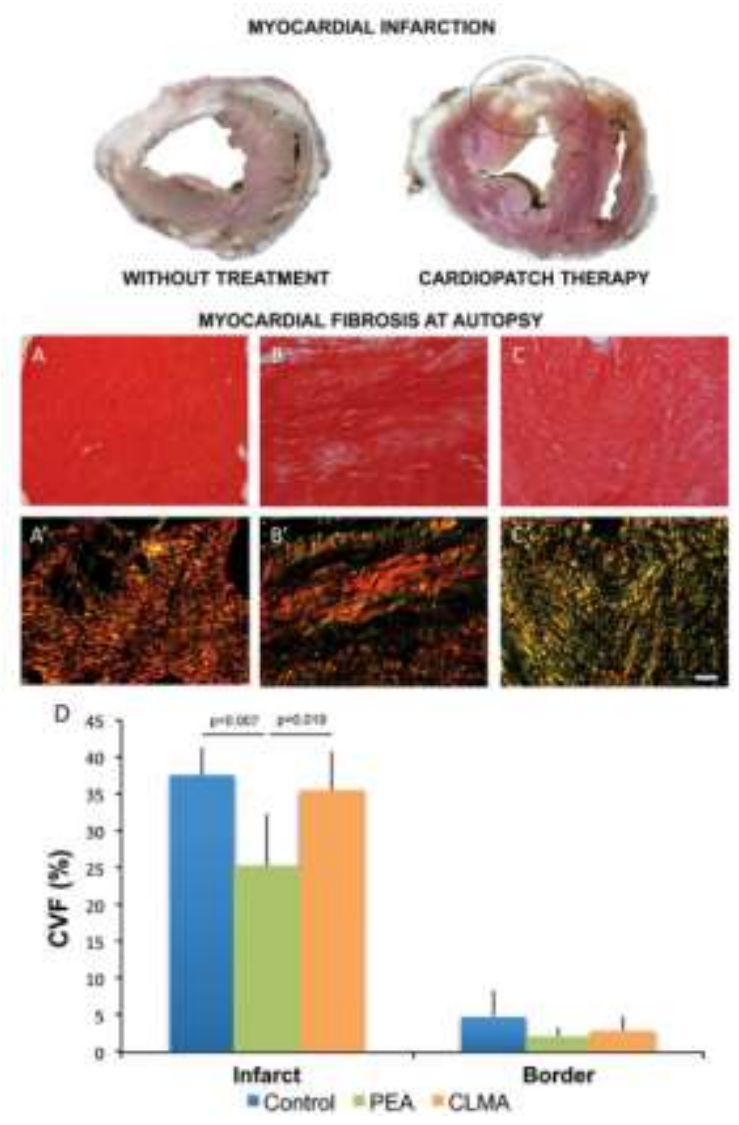


Figure 3: Macroscopic pathology of sheep ventricles showing white scars of myocardial infarction at 6 months. (Upper left) Control untreated heart with large infarct scar. (Upper right) Reduced size of infarct scar in sheep treated with CLMA cardiopatch (encircled area). (Lower pictures) Evaluation of myocardial fibrosis at autopsy. Sirius red staining distinguishing collagen (red) in infarct zones from control (A), CLMA (B) and poly(ethyl) acrylate (C) groups. ( $\left.A^{\prime}-C^{\prime}\right)$ Polarized light microscopy images exhibit collagen I (red/yellow) and collagen III (green) fibrils in the same sections. Scale bar $50 \mathrm{~mm}$. (D) CVF in percentage measured on polarized light images. Results are presented as the mean percentage of CVF. Error bars: \pm standard deviation. P-value $<0.05$ was considered significantly different. CLMA: cross-linked polycaprolactone; CVF: collagen volume fraction; PEA: poly(ethyl) acrylate.

Expression of MMP9 was also analysed due to its possible role in the degradation of ECM and in myocardial remodelling. Immunostaining against MMP9 in all the experimental groups revealed the presence of MMP9-positive inflammatory cells in infarct, border and remote zones (Fig. 4). Animals treated with the PEA membrane had a statistically significantly lower quantity of MMP9-expressing cells in border and remote areas $(P<0.001$ and $P=0.005$, respectively). CLMA-treated animals, however, presented with a higher number of MMP9positive cells in the border zone $(P=0.002)$ (Fig. 4).

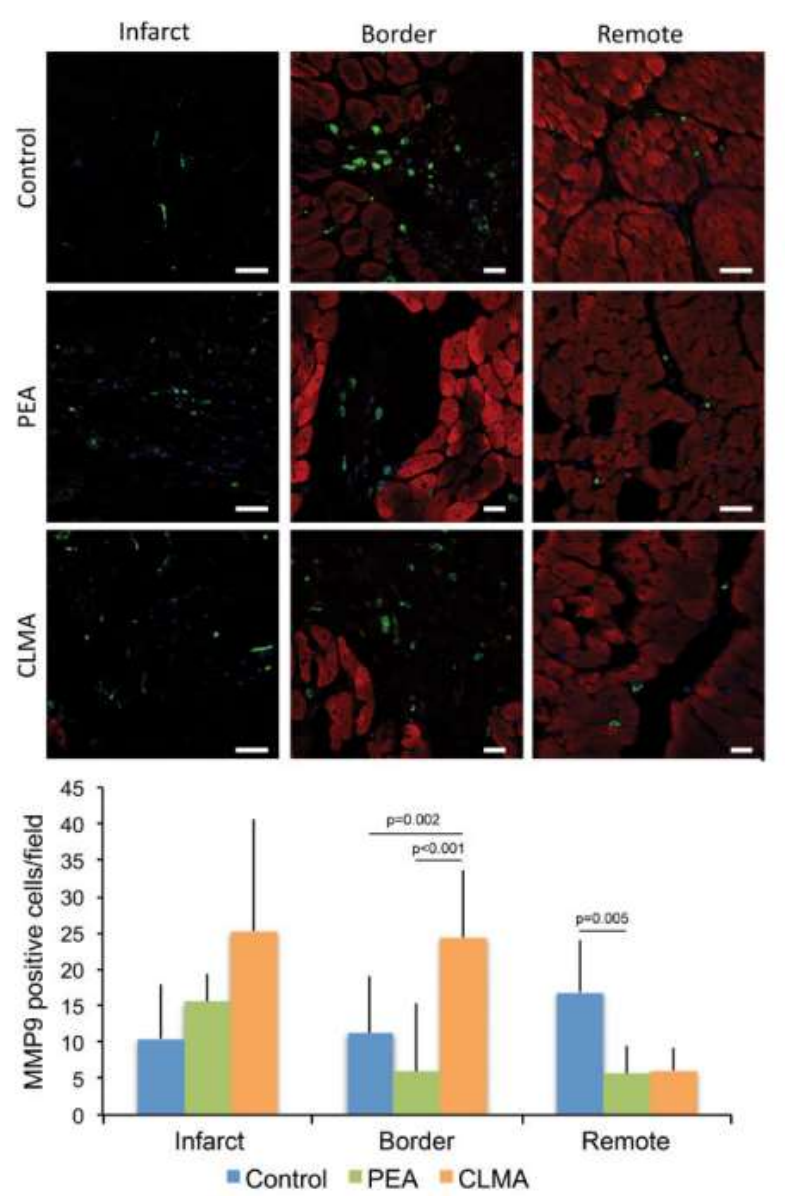

Figure 4: Immunodetection of MMP9 in control, PEA and CLMA-treated animals. MMP9 (green), cardiac troponin I (red) and nuclei (40,6-diamidino-2-phenylindole, blue). Scale bars $50 \mathrm{~mm}$. Histogram of the number of MMP9positive cells per field in infarct, border and remote zones. Results are presented as the mean number of MMP9positive cells per field \pm standard deviation. P-value $<0.05$ was considered significantly different. CLMA: cross-linked polycaprolactone; MMP9: metalloproteinase 9; PEA: poly(ethyl) acrylate. 
ATDPCs positive for RFP were found inside the patch and in the infarct scars and healthy myocardium in both treated groups (Fig. 5A-H). Hence, ATDPCs were able to migrate from the cardiopatch to the nearby tissue (Fig. 5A and E). Integration of ATDPCs into the vessel structures was also observed in the cardiopatch interconnected with myocardium (Fig. 5B and F). ATDPCs therefore contributed to the formation of a capillary network between the cardiopatch and the myocardium (Fig. 5C, D, G and H).
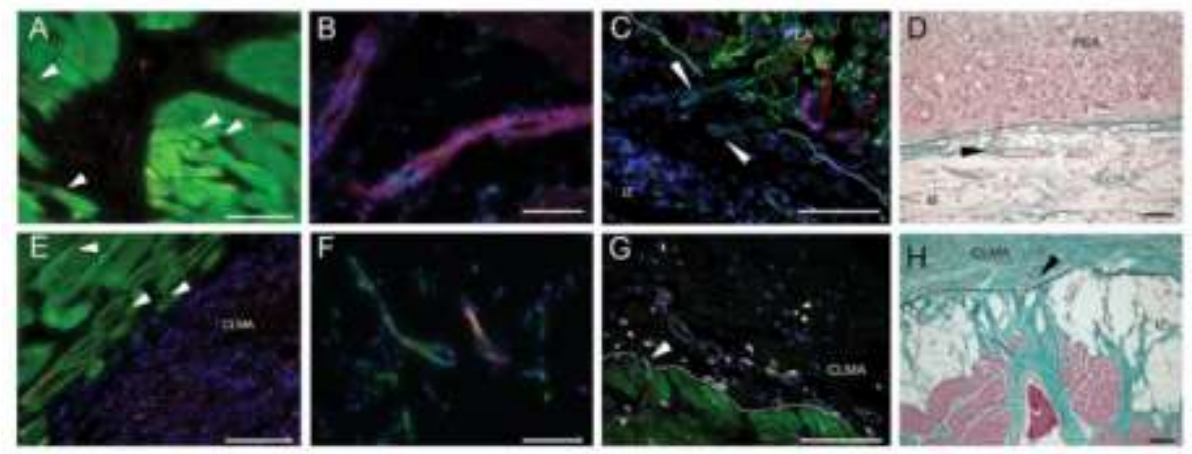

Figure 5: Immunostaining of heart sections. Sheep infarcted hearts treated with adipose tissue-derived progenitor cells (ATDPCs) (red) loaded in PEA (A-D) or CLMA cardiopatches (E-H). (A and E) Presence of ATDPCS (red, arrowheads) in the myocardium [cardiac troponin I (cTnl), green]. (B, C and F) Presence of vessels (green) in the cardiopatches and integration of ATDPCs in the vessels (magenta). (G) Vessels (white), cTnl (green) and ATDPCs (red) staining. Nuclei (40,6-diamidino-2-phenylindole, blue). ( $D$ and $H$ ) Masson's trichrome staining of heart sections. Arrowheads in C, D, G and $\mathrm{H}$ indicate vascular connections. Scale bars $100 \mathrm{~mm}$ in A-C, E-G and $200 \mathrm{~mm}$ in D and H. CLMA: cross-linked polycaprolactone; IZ: infarcted zone; PEA: poly(ethyl) acrylate.

Evaluation of vessel density revealed a greater percentage of vessels in the infarcted areas in animals treated with CLMA patches compared to the controls and the PEA-treated groups (1.17 $\pm 0.27,0.70 \pm 0.09$ and $0.68 \pm 0.09$, respectively) with statistically significant differences (CLMA vs controls, $\mathrm{P}=0.025$; CLMA vs PEA, $\mathrm{P}=0.019$ ) (Fig. 6). No differences in vessel density were observed between the controls and the PEA-treated animals in infarct zones or in border myocardium. These results suggest that CLMA patches, together with implanted ATDPCs, may promote the formation of new vessels in the ischaemic myocardium.

At the level of the epicardium, we found a minimal fibrosis interface without inflammation between both the cardiopatch and the heart surface. The CLMA patch was completely anchored to and incorporated into the nearest infarct area and the myocardium. In contrast, the PEA patches macroscopically showed poor adhesions to the heart and the infarct scar although some areas were nicely attached to the myocardium (Fig. 5D). Overall, the bioabsorbable CLMA patches possessed improved flexibility and adapted better to the curvature of the heart's surface than did the biostable hydrophobic PEA patches. 


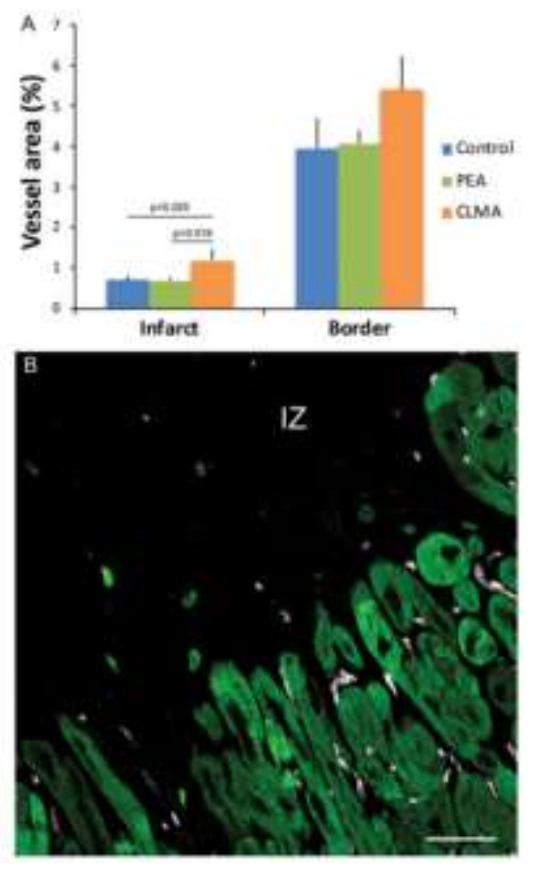

Figure 5: Immunostaining of heart sections. Sheep infarcted hearts treated with adipose tissue-derived progenitor cells (ATDPCs) (red) loaded in PEA (A-D) or CLMA cardiopatches (E-H). (A and E) Presence of ATDPCS (red, arrowheads) in the myocardium [cardiac troponin I (cTnl), green]. (B, C and F) Presence of vessels (green) in the cardiopatches and integration of ATDPCs in the vessels (magenta). (G) Vessels (white), cTnl (green) and ATDPCs (red) staining. Nuclei (40,6-diamidino-2-phenylindole, blue). ( $D$ and $H$ ) Masson's trichrome staining of heart sections. Arrowheads in C, D, G and $H$ indicate vascular connections. Scale bars $100 \mathrm{~mm}$ in A-C, E-G and $200 \mathrm{~mm}$ in D and H. CLMA: cross-linked polycaprolactone; IZ: infarcted zone; PEA: poly(ethyl) acrylate.

\section{DISCUSSION}

Results of isolated stem cell therapy in cardiac diseases showed that cell bioretention and engraftment within infarcts are low because cell homing is limited by the degradation of the ECM, resulting in adverse LV remodelling $[2-4,13]$. The association of elastomeric scaffolds with stem cells raises the possibility of repairing the damaged myocardial tissue and avoiding ventricular chamber dilation. The cardiopatch seems appropriate to support $\mathrm{MI}$ scars by a 'Band-Aid' effect, limiting the spread of the infarcted areas and improving the ventricular wall by minimizing stress tolerance by a girdling effect [7].

Cardiowrap-biological ventricular support The first biomechanical strategy limiting ventricular dilatation was the latissimus dorsi dynamic-cardiomyoplasty for patients with severe heart failure awaiting a heart transplant [14]. More than 2000 patients have been operated on worldwide; cardiomyoplasty provides an autologous source of circulatory assistance. The chronically electrostimulated latissimus dorsi skeletal muscle flap wrapped around the heart works in concert with the myocardium, improving the haemodynamics via an electronic cardiomyostimulator device with electrodes. Less invasive alternative approaches like ventricular restraint therapy using polyester mesh wraps and nitinol devices have been proposed for patients with heart failure; however, these constraint acellular devices have failed to demonstrate clear clinical benefits [15]. A new approach based on myocardial tissue engineering might instead herald myocardial healing and evidence of ventricular support [5]. 
The main purpose of the cardiopatch is to display structural and functional properties similar to those of natural extracellular matrices containing 3D nanostructures and proper instructive niches for cell homing [8-10]. Speckle-tracking ultrasound revealed positive results about deformation of the LV and improvements in patients with MI treated with a cardiopatch versus a control group. MRI analysis of the infarct area using serial planes showed significant reduction of the infarct scar in the cardiopatch-treated groups [11, 12]. Histopathologically, matrix metalloproteinases are endopeptidases that cleave all components of the ECM and participate in LV remodelling [16]. In particular, MMP9 is important because upregulation after the $\mathrm{MI}$ and gene deletion attenuate LV remodelling [17]. Furthermore, studies of infarct rupture have also demonstrated that MMP9 is expressed by inflammatory cells in the infarcted area [17]. The biodegradable characteristics of the CLMA cardiopatch may induce a greater inflammatory effect, thereby increasing the number of MMP9-positive cells. Thus, the elevated density of the vessel may facilitate cell mobilisation in an MI treated with CLMA. The presence of ATDPCs in the scar and in the healthy myocardium seems to indicate that the implanted ATDPCs are able to migrate from the cardiopatch to the nearby cardiac tissue, possibly promoting angiogenesis. This outcome was suggested in a previous study in which human ATDPCs loaded in CLMA patches expressed cardiac troponin I when implanted in a mouse model of MI [10]. Complete vascularization of implanted CLMA and PEA patches was also demonstrated in addition to vascular connections in the myocardial-cardiopatch interface. This finding is important for cell survival and for the proper integration of the bioactive implant over the cardiac tissue. The composition of the patches and the peptide hydrogel RAD16-I facilitated capillary formation and distribution, as was previously experimentally shown in vitro [18]. Interestingly, ATDPCs were integrated in some of those vessels, thereby contributing to the capillary network. This finding was also observed in human ATDPCs implanted via a fibrin patch in a murine MI model [19]. Analysis of vascular density revealed an increase in the percentage of the vascular area in the infarcted zones when animals were treated with CLMA patches and ATDPCs compared to those treated with PEA and the controls. The channelled structure and progressive biodegradation characteristics of the CLMA patches might facilitate the diffusion of the proangiogenic factors from the patch to the myocardium more easily than the PEA biostable patches.

\section{Clinical translation}

The first clinical application of in vivo myocardial tissue engineering was the MAGNUM (Myocardial Assistance by Grafting a New bioartificial Upgraded Myocardium) trial in 2007. In this study, a collagen type I cardiopatch seeded with mononuclear bone marrow cells was grafted onto LV MI scars [20]. In this example of in vivo tissue engineering, the body is the bioreactor. The lack of adequate tensile properties and the lack of controlled bioresorption of collagen cardiopatch boosted our interest for the use of elastomer scaffolds and nanofibres, creating a cardiopatch-like bioartificial myocardium. This novel cardiac repair strategy included a porous elastomeric 3D polycaprolactone cardiopatch filled with Puramatrix-containing stem cells, which mimics the biophysical and biomechanical attributes of natural myocardium. Our study demonstrates the feasibility and effectiveness of a cardiopatch grafted onto MI scars in large animals. This treatment reduced infarct volume and fibrosis, limited ventricular deformation and improved ventricular function. There was minimal fibrosis interface with minimal inflammation between the heart and the patch. The cardiopatch was firmly anchored 
and integrated to the infarct area and the myocardium; it promoted new vessel formation in ischaemic myocardium; it reduced the number of inflammatory cells expressing MMP9; and, therefore, reduced adverse cardiac remodelling and the risk of postinfarct ventricular rupture $[16,17]$.

\section{Perspectives}

Cardiopatch biotechnology might be applied to the creation of cardiowrap bioprostheses for external ventricular support with myocardial repair, thereby reducing progression to heart failure and the indication for a heart transplant. The proposed ventricular support bioprostheses are manufactured with elastomeric polycaprolactone membranes (helical bands) to avoid progression of adverse ventricular remodelling (see Supplementary data: Invention Patents Chachques et al. 'Bioactive implants for myocardial regeneration and ventricular chamber restoration': US Patent 8968417 B2, 2015; US Patent Part2 10004602 B2, 2018; European Patent 2422823 B1, 2014). The design of the ventricular support bioprostheses is based on the concept of the 'helical myocardial band', which describes the anatomical configuration of the heart, whereby muscular ventricular bands provide conical configuration to the LV chamber (Torrent-Guasp concept). The role of the myocardial band is to limit ventricular dilatation, preserving elliptical shape and contributing to systolic contraction and diastolic filling (suction mechanism). A ventricular support bioprosthesis is used to wrap the ventricles, starting near the left atrial appendage at the level of the pulmonary artery root and ending at the level of the aortic root. The loop/band can be affixed onto the heart and/or onto the cardiopatch by surgical sutures, biological or synthetic glue or surgical clips. Future cardiowrap scaffolds might be manufactured using 3D printing [21] and new functionalization strategies, focusing on nanocontainer technology for the sustained release of active molecules (basic fibroblast growth factor, vascular endothelial growth factor, epidermal growth factor). The nanocontainers are fixed onto poly(e-caprolactone) fibres, which improve tissue repair [22]. Another approach to enhance the bioactivity of the scaffolds is to graft poly(sodium styrene sulfonate) onto poly(ecaprolactone) membranes [23]. The implantation of a poly-Llactide granulocyte colony-stimulating factor-functionalized scaffold in a rabbit model of chronic $\mathrm{MI}$ induced an angiogenetic process and the reorganization of the ECM architecture leading to connective tissue deposition and scar remodelling [24].

\section{Limitations}

To avoid the risk of death related to exposure to general anaesthesia and heart manipulation during dissection of the infarct scar area, the animals in group 1 did not have a sham operation at 2 months. Our surgical MI was created by ligation of diagonal branches of the left anterior descending artery. This procedure results in a segmental dysfunction of the anterior and lateral walls without malignant ventricular arrhythmias, thereby avoiding massive LV failure, which results in a high operative mortality rate in sheep [25]. Surgical induction of infarcts in the diagonal area of the LV also may not be clinically relevant because of the small infarct territory. Moreover, catheter-based MI models are still associated with a high mortality rate [25]. In accordance with the regulations of our university ethics committee, research projects using largeanimal models must strictly abide by the rules related to the minimum necessary number of groups and interventions to evaluate new surgical procedures and to satisfy 
statistical requirements. The MRI system available in our research centre only allowed evaluation of small animals (rabbit, rats, mice). For this reason, we performed ex vivo MRI evaluation of the sheep hearts at 6 months to measure MI volumes. Procedures included removing the hearts after the last echocardiographic assessment and $15 \mathrm{~min}$ after administering intravenous injections of gadolinium. The sheep were given general anaesthesia and an overdose of the anaesthetic pentobarbital.

\section{CONCLUSION}

In summary, we were able to successfully integrate the cardiopatch histologically into damaged myocardium and adjacent to healthy tissue, such that it became an artificial ECM that offered adequate cell niches for the homing of stem cells or exosomes [26]. Results from this study substantially contributed to the generation of an elastomeric cardiopatch and opened the way to create cardiowrap-supported bioprostheses that may become clinically applicable in the supportive treatment of ischaemic heart disease and chronic heart failure [1, $5,6,15]$.

\section{ACKNOWLEDGEMENTS}

The authors acknowledge networking support from the EU COST Action CA16122. Funding The RECATABI Project (Regeneration of Cardiac Tissue Assisted by Bioactive Implants) was financially supported by the 7th Framework Programme (FP7) of the European Commission. Project ID: 229239. Funded under FP7-NMP and the European Regional Development Fund (FEDER Spain).

Conflict of interest: none declared.

\section{REFERENCES}

[1] Madonna R, Van Laake LW, Botker HE, Davidson SM, De Caterina R, Engel FB et al. ESC Working Group on Cellular Biology of the Heart: position paper for Cardiovascular Research: tissue engineering strategies combined with cell therapies for cardiac repair in ischemic heart disease and heart failure. Cardiovasc Res 2019;115:488-500.

[2] Nielsen SH, Mouton AJ, DeLeon-Pennell KY, Genovese F, Karsdal M, Lindsey ML. Understanding cardiac extracellular matrix remodeling to develop biomarkers of myocardial infarction outcomes. Matrix Biol 2019;75-76:43-57.

[3] Spinale FG, Frangogiannis NG, Hinz B, Holmes JW, Kassiri Z, Lindsey ML. Crossing into the next frontier of cardiac extracellular matrix research. Circ Res 2016;119:1040-5.

[4] Vu DT, Martinez EC, Kofidis T. Myocardial restoration: is it the cell or the architecture or both? Cardiol Res Pract 2012;2012:240497.

[5] Chachques JC, Pradas MM, Bayes-Genis A, Semino C. Creating the bioartificial myocardium for cardiac repair: challenges and clinical targets. Expert Rev Cardiovasc Ther 2013;11:170111. 
[6] Bayes-Genis A, Galvez-Monton C, Roura S. Cardiac tissue engineering: lost in translation or ready for translation? J Am Coll Cardiol 2016;68: 724-6.

[7] Shafy A, Fink T, Zachar V, Lila N, Carpentier A, Chachques JC. Development of cardiac support bioprostheses for ventricular restoration and myocardial regeneration. Eur J Cardiothorac Surg 2013;43: 1211-19.

[8] Castells-Sala C, Recha-Sancho L, Lucía-Valldeperas A, Soler-Botija C, Bayes-Genis A, Semino CE. Three-dimensional cultures of human subcutaneous adipose tissue-derived progenitor cells based on RAD16-I self-assembling peptide. Tissue Eng Part C Methods 2016;22:113-24.

[9] Martínez-Ramos C, Rodríguez-Pérez E, Garnes MP, Chachques JC, Moratal D, Valle' s-Lluch $A$ et al. Design and assembly procedures for large-sized biohybrid scaffolds as patches for myocardial infarct. Tissue Eng Part C Methods 2014;20:817-27.

[10] Soler-Botija C, Bago' JR, Llucia` -Valldeperas A, Vallés-Lluch A, CastellasSala C, Martı́nezRamos $C$ et al. Engineered 3D bioimplants using elastomeric scaffold, self-assembling peptide hydrogel, and adipose tissuederived progenitor cells for cardiac regeneration. Am J Transl Res 2014; 6:291-301.

[11] Biswas M, Sudhakar S, Nanda NC, Buckberg G, Pradhan M, Roomi AU et al. Two- and three-dimensional speckle tracking echocardiography: clinical applications and future directions. Echocardiography 2013;30: 88-105.

[12] Dorsey SM, McGarvey JR, Wang H, Nikou A, Arama L, Koomalsingh KJ et al. MRI evaluation of injectable hyaluronic acid-based hydrogel therapy to limit ventricular remodeling after myocardial infarction. Biomaterials 2015;69:65-75.

[13] Chachques JC. Cellular cardiac regenerative therapy in which patients? Expert Rev Cardiovasc Ther 2009;7:911-19.

[14] Chachques JC, Marino JP, Lajos P, Zegdi R, D'Attellis N, Fornes $P$ et al. Dynamic cardiomyoplasty: clinical follow-up at 12 years. Eur J Cardiothorac Surg 1997;12:560-7.

[15] Varela CE, Fan Y, Roche ET. Optimizing epicardial restraint and reinforcement following myocardial infarction: moving towards localized, biomimetic, and multitherapeutic options. Biomimetics (Basel) 2019;4:7.

[16] Van den Borne SW, Cleutjens JP, Hanemaaijer R, Creemers EE, Smits JF, Daemen MJ et al. Increased matrix metalloproteinase- 8 and -9 activity in patients with infarct rupture after myocardial infarction. Cardiovasc Pathol 2009;18:37-43.

[17] Ducharme A, Frantz S, Aikawa M, Rabkin E, Lindsey M, Rohde LE et al. Targeted deletion of matrix metalloproteinase-9 attenuates left ventricular enlargement and collagen accumulation after experimental myocardial infarction. J Clin Invest 2000;106:55-62.

[18] Sieminski AL, Semino CE, Gong H, Kamm RD. Primary sequence of ionic selfassembling peptide gels affects endothelial cell adhesion and capillary morphogenesis. J Biomed Mater Res Part Res 2008;87:494-504. 
[19] Bago' JR, Soler-Botija C, Casanı' L, Aguilar E, Alieva M, Rubio N et al. Bioluminescence imaging of cardiomyogenic and vascular differentiation of cardiac and subcutaneous adipose tissue-derived progenitor cells in fibrin patches in a myocardium infarct model. Int J Cardiol 2013;169:288-95.

[20] Chachques JC, Trainini JC, Lago N, Cortes-Morichetti M, Schussler O, Carpentier A. Myocardial Assistance by Grafting a New Bioartificial Upgraded Myocardium (MAGNUM trial): clinical feasibility study. Ann Thorac Surg 2008;85:901-8.

[21] Lee H, Ahn S, Bonassar L, Kim G. Cell(MC3T3-E1)-printed poly(e-caprolactone)/alginate hybrid scaffolds for tissue regeneration. Macromol Rapid Commun 2013;34:142-9.

[22] Strub M, Van Bellinghen X, Fioretti F, Bornert F, Benkirane-Jessel N, Idoux-Gillet $Y$ et al. Maxillary bone regeneration based on nanoreservoirs functionalized e-polycaprolactone biomembranes in a mouse model of jaw bone lesion. Biomed Res Int 2018;2018:7380389.

[23] Rohman G, Huot S, Vilas-Boas M, Radu-Bostan G, Castner DG, Migonney V. The grafting of a thin layer of poly(sodium styrene sulfonate) onto poly(e-caprolactone) surface can enhance fibroblast behavior. J Mater Sci Mater Med 2015;26:5539.

[24] Spadaccio C, Nappi F, De Marco F, Sedati P, Taffon C, Nenna A et al. Implantation of a Poly-L-Lactide GCSF-Functionalized Scaffold in a model of chronic myocardial infarction. J Cardiovasc Trans Res 2017;10: 47-65.

[25] Monnet E, Chachques JC. Animal models of heart failure: what is new? Ann Thorac Surg 2005;79:1445-53.

[26] Bellin G, Gardin C, Ferroni L, Chachques JC, Rogante M, Mitrecic D et al. Exosome in cardiovascular diseases: a complex world full of hope. Cells 2019;8:166. 